\title{
Methodology for the nuclear design validation of an Alternate Emergency Management Centre (CAGE)
}

\author{
César Hueso ${ }^{1}$, Marco Fabbri ${ }^{1,3}$, Cristina de la Fuente ${ }^{1}$, Albert Janés ${ }^{1}$, Joan Massuet ${ }^{1}$, Imanol Zamora ${ }^{1}$, Cristina Gasca ${ }^{2}$, \\ Héctor Hernández ${ }^{2}$, J. Ángel Vega ${ }^{2}$ \\ ${ }^{1}$ Idom Ingeniería y Consultoría, Avda. Zarandoa, 23 Bilbao-Vizcaya-Spain 48015, +34 944797600 \\ ${ }^{2}$ ANAV Asociación Nuclear Ascó-Vandellòs II, L'Hospitalet de l'Infant-Tarragona-Spain 43890, +34 977818800 \\ ${ }^{3}$ Fusion for Energy, C/Josep Pla, 2, Torres Diagonal Litoral, Edif. B3 Barcelona-Spain 08019, +34 933201800
}

\begin{abstract}
The methodology is devised by coupling different codes. The study of weather conditions as part of the data of the site will determine the relative concentrations of radionuclides in the air using ARCON96. The activity in the air is characterized depending on the source and release sequence specified in NUREG1465 by RADTRAD code, which provides results of the inner cloud source term contribution. Known activities, energy spectra are inferred using ORIGEN-S, which are used as input for the models of the outer cloud, filters and containment generated with MCNP5. The sum of the different contributions must meet the conditions of habitability specified by the CSN (Spanish Nuclear Regulatory Body) (TEDE $<50 \mathrm{mSv}$ and equivalent dose to the thyroid $<500 \mathrm{mSv}$ within 30 days following the accident doses) so that the dose is optimized by varying parameters such as CAGE location, flow filtering need for recirculation, thicknesses and compositions of the walls, etc. The results for the most penalizing area meet the established criteria, and therefore the CAGE building design based on the methodology presented is radiologically validated.
\end{abstract}

\section{Introduction}

After the earthquake and tsunami on March 11, 2011 in Fukushima Dai-chi, all nuclear plants in the European Union have been subjected to "stress tests". The Spanish nuclear sector has proposed, and the CSN has subsequently required, the creation of a centre to safely manage an emergency, called Alternate Emergency Management Centre (CAGE) located at the sites of the Nuclear Power Plants [Ref. 1]. Living conditions of the occupants of the CAGE in the event of a Severe Accident imply that TEDE must be $<50 \mathrm{mSv}$ and the equivalent dose to the thyroid is $<500 \mathrm{mSv}$ within 30 days following the accident [Ref. 2]. Given the weather conditions of each plant, the calculations are analogous to those supporting the Control Room and the different ways of radiation exposure or contamination are simulated [Fig. 1]. These paths that contributes to the dose are:

- Determination of dose due to inner radioactive cloud (within the CAGE).

- Determination of dose due to the presence of the radioactive cloud outside the CAGE.

\begin{abstract}
- Determination of dose due to the accumulation of radionuclides in the filters.

- Determination of dose due to the proximity to the containment.

The variety of contributions to the dose has to be approached in an integral way. Each contribution is due to a different source term or a different interaction with the human body (i.e. external exposure, internal contamination, etc.) that have to be taken into account. Considering that a radioactive cloud stands around the CAGE during the duration of the accident ( 720 hours), different situations arises.

Regarding the consequences of radioactive materials being incorporated in the inside CAGE atmosphere, external exposure and inhalation of radionuclides contributions have to be evaluated. This contribution requires the knowledge of the radiation transport mechanism and of the site meteorological data. To help solving this problem, the ARCON96, (Atmospheric Relative Concentration in building wakes) [Ref. 3] and RADTRAD 3.03 [Ref. 4] are applied.
\end{abstract}

\footnotetext{
* Corresponding author: cesar.hueso@idom.com
} 


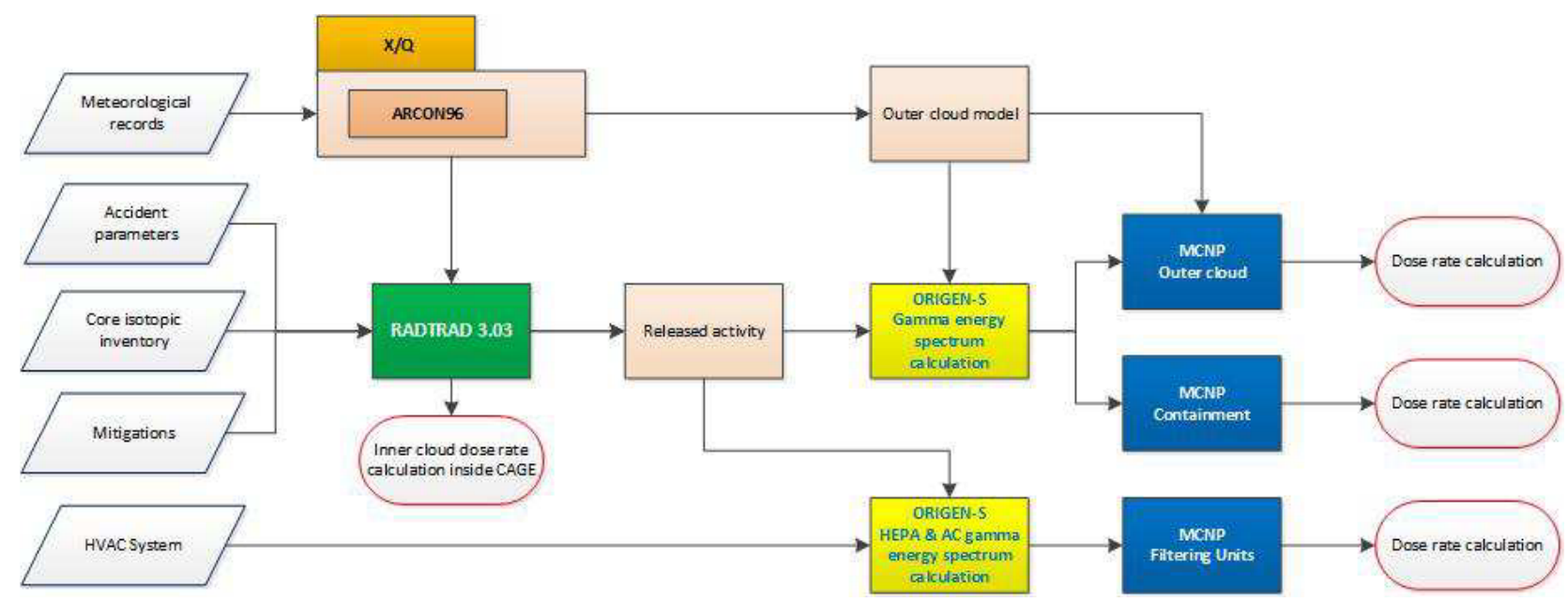

Fig. 1. Methodology applied to determine different dose contributions.

On the other hand, the fact of the radioactive cloud standing around the CAGE becomes a shielding problem where the source term is outside and the people to protect is inside. Therefore, a shielding has to be designed, mainly the concrete walls and doors. After assuming a geometry and applying the radionuclides activity released to the environment (RADTRAD), ORIGEN-S [Ref. 5] is used to "translate" the activities into gamma radiation energy spectra. These spectra are introduced as input data in a Monte Carlo radiation transport calculation by means of MCNP [Ref. 6]. This code delivers the outer cloud contribution.

In a similar way, the containment direct radiation is assessed. The only difference is that in this case, the inside containment activities (RADTRAD) are considered. This problem is highly demanding from a computational point of view because of the thicknesses of shielding (containment and CAGE concrete walls) and distance involved.

And last but not least, the outer radioactive cloud is being filtered by the filtering units. These HEPA and active carbon filters are not perfect and inner cloud contribution is due to their small inefficiency. Nevertheless the most of the radionuclides are accumulated as the filters work, resulting in a strong source term. To perform this calculation, the activities of filtered radionuclides, and their daughter's activities, are taken into consideration. Again thanks to ORIGEN-S, these activities are "translated" into gamma energy spectra to be introduced as input data in a new radiation transport calculation that delivers the dose contribution due to the filtering units.

\section{Assumptions and Input data}

To carry out the necessary calculations by coupling the various codes that perform the methodology used to determine dose rates, there have to be considered situations and initial data that will determine the suitability of the resulting solutions. Then the input data and assumptions depending on the location of the CAGE are presented, as well as for each of the contributions to the final dose rate.

\subsection{Diffusion factors}

According to RG (Regulatory Guide) 1.23 Rev. 1 [Ref. 7], a Nuclear Power Plant should be able to get the weather information it requires to, among other objectives, determine the potential spread of radioactive material from an accident, so it can be deducted the amount of radionuclides resulting from the release into the environment of the considered source term. The ARCON96, it is a tool developed by the Nuclear Regulatory Commission to perform calculations of diffusion factors for habitability analysis of Control Rooms of Nuclear Power Plants in compliance with RG 1.194 [Ref. 8].

The following structure summarizes the different steps carried out to calculate the atmospheric relative concentrations $(\mathrm{X} / \mathrm{Q})$ of radioisotopes:

1.Obtaining meteorological data.

2.Process meteorological data.

- Calculation of the hourly averages.

- Calculation of atmospheric stability. 
- Generation of meteorological files for ARCON96.

3.ARCON96 execution.

\subsection{Obtain and process meteorological data}

Weather information is provided by specific files, including the matrix of hourly frequencies, defined from the following time averages:

- Wind speed (in $\mathrm{m} / \mathrm{s}$ ) at different heights.

- Wind direction (in degrees) at different heights.

- Category stability (Pasquill, from A to G).

\subsubsection{Hypothesis}

- RG 1.194 considers representative hourly weather observations for more than 5 years.

- Height measurement data at $10 \mathrm{~m}$ and $29 \mathrm{~m}$.

- An emission at ground level is assumed; conservative assumptions at the selected location distance.

- Conservatively, a height equal to intake $0 \mathrm{~m}$ is assumed.

- A 'terrain elevation difference' is taken equal to $0 \mathrm{~m}$, since no data are available about it.

- Building line perpendicular to the direction of the release section.

- The angle between the CAGE and the emission source, considering not to locate the building in a predominant wind window.

- 90 degree wind window is taken.

- Distance from the emission point to CAGE: measured on the ground.

- Minimum wind speed $0.5 \mathrm{~m} / \mathrm{s}$.

- Surface roughness of $0.20 \mathrm{~m}$.

- The initial values of $\sigma_{\mathrm{y}}$ and $\sigma_{\mathrm{z}}$ are equal to 0 , as advised in RG 1.194.

\subsection{Determination of dose}

Once the diffusion factors have been obtained and therefore, the relative concentration of radionuclides known in the points to study, the analysis of the different routes of contribution to the dose within 30 days of accident principles is studied.

The aforementioned diffusion factors will be introduced as input data in the codes to be used for calculations of radiation transport.

\subsubsection{Inner cloud contribution. Input data and assumptions}

Determining dose inside the cloud will take place through software RADTRAD, as shown in Figure 1, since as specified in NUREG-1465 [Ref. 9] and the RG 1195 [Ref. 10] it is a code that incorporates adequate methodologies to meet dose determination.

Then the necessary data and hypotheses considered are as follows:

- Diffusion factors or X/Q obtained through the ARCON96 code.

- Flow diagram of HVAC system.
- Decontamination factor by natural deposition of elemental iodine.

- Chemical composition of radio-iodine, extracted value from NUREG-1465.

- Containment volume.

- Thermal power of the reactor.

- Reactor core inventory, it was assumed to be consistent with the inventory from the post-Fukushima stress test project.

- Overpressure flow calculation to define the radiological classification of CAGE.

- Net volumes of each of the areas of the CAGE.

- FGR 11: Limit Values of Radionuclide Intake and Air concentration and Dose Conversion Factors for Inhalation, Submersion, and Ingestion. 1988 [Ref. 11].

- FGR 12: External Exposure to Radionuclides in Air, Water and Soil [Ref. 12].

Furthermore, in regard to assumptions, the following are considered:

- Two different zones are considered in the CAGE. Zone $A$, which will be in excess of pressure compared to Zone B. Note that both of them are in overpressure from the atmospheric pressure.

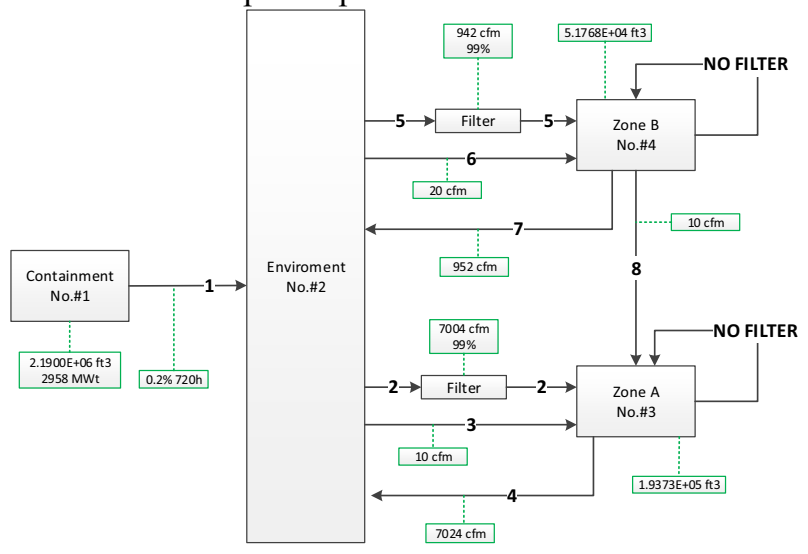

Fig. 2. Model RADTRAD.

- Release rates in the event of a severe accident in reactors PWR / BWR, are introduced into the RADTRAD code. The severe accident definition is in line with the post-Fukushima stress test accident definition. Note that the release fraction and timings for severe accident in RADTRAD are the ones from NUREG-1465, Tables 3.12 and 3.13.

- Isotopes of the source term are introduced by the corresponding external .nif file.

- Loading factors defined in RG 1.195 are used.

- Breathing rates according RG 1.195 , being $3.5 \mathrm{E}^{-4} \mathrm{~m}^{3} / \mathrm{s}$ at 720 hours.

- Credit is given to radioactive decay.

- Inflow of air and recirculation values established by the HVAC system are set for Zone A and B.

- In the compartment defined as containment credit is given to the natural deposition.

- A release of radionuclides to the environment is estimated corresponding to $0.2 \%$ of the containment volume per day during the 30 days of the accident postulated, not only during the first 24 hours to add conservatism to the calculations. 
- Outside contaminated air inlet is considered to be filtered by HEPA and active carbon filtering units.

- Infiltrations flow is introduced from the outside contaminated environment into Zone A $10 \mathrm{cfm} /$ Zone B $20 \mathrm{cfm}$ in line with the RG 1.78 [Ref. 13].

- The discharge rate compensates for infiltrations and the supply flow.

- Infiltrations flow from Zone B to Zone A $10 \mathrm{cfm}$.

- Several time steps are considered for evaluation according to the diffusion factor or $\mathrm{X} / \mathrm{Q}$.

\subsubsection{External cloud contribution. Input data and assumptions}

Determining dose provided by the outer cloud to CAGE is carried out by coupling software RADTRAD 3.03, ORIGEN-S and MCNP as indicated in the flow diagram of Figure 1. RADTRAD 3.03 was used for estimating the release of radioactive materials into the environment in case of a severe accident. Then, using the diffusion factor determined by ARCON96, the average isotopic activity contained in the radioactive cloud in which is immersed the CAGE is obtained. Likewise, the corresponding gamma energy spectrum is determined by software ORIGEN-S, in which are entered as input data the activities obtained for each time interval. Finally, these gamma spectra are introduced in their respective MCNP5 simulations in order to characterize the cloud corresponding to the outer volume source. It is also needed:

- Simplified but representative model the geometry of the CAGE.

- Modeling the radioactive cloud as a semi-cylindrical source.

- Defining the parameters of interest of the simulation and the respective location where these values are extracted.

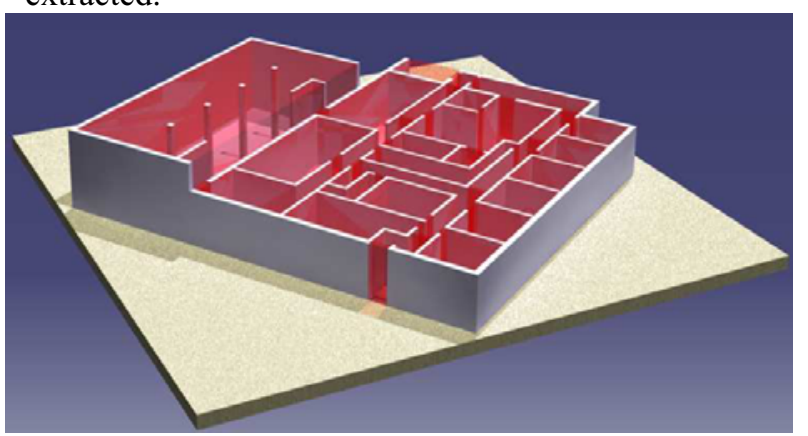

Fig. 3 Simplified geometry CAGE

Therefore, as input data are considered:

- Isotopic activity released to the outside environment.

- Diffusion factors or X/Q obtained through ARCON96 code.

- Figures 4 and 5 of RG 1.194 are used for the determination of the diffusion coefficients $\sigma_{\mathrm{z}}$ and $\sigma_{\mathrm{y}}$. Note that once all the parameters from equation 1 form section 3.2 of the NUREG/CR6331 [Ref. 3] are known, the distance from the center of the plume (i.e. parameter $y$ from the equation 1 of the present paper) can be calculated for each time step. This y parameter allows for the cloud volume definition.
- It is considered that the external environment is in the stability class of type $G$ since thereby stability coefficients are minimized.

- "Still air" speed considered, being $0.5 \mathrm{~m} / \mathrm{s}$.

- Project drawings for the determination of the simplified geometry of CAGE.

- Gamma energy spectra for the characterization of the corresponding to the outer cloud over the CAGE volume source.

- Photon libraries MCPLIB84 included in the MCNP5 package.

- Radiological properties of the materials considered in the CAGE.

And the main assumptions are as follows:

- For simplistic effects it has been assumed that the cloud over the CAGE has a semi-cylindrical geometry. Therefore,

$$
V_{\text {cloud }}=1 / 2 \pi y^{2} L
$$

$V_{\text {cloud }}(m 3)$

$y(m)$

$L(m)$

- At each time step, a uniform concentration is assumed.

- Significantly, it is considered that the transport of the release to the CAGE is instantaneous.

- The most representative concrete walls of the CAGE are modeled.

- CAGE slabs and the outer ground to take into account the backscattering are modeled.

- No accumulation of radioisotope within the CAGE.

- The contribution of the neutron dose determination is neglected.

- In each time step, the intensity corresponding to the selected volume source spectrum previously calculated by ORIGEN-S is assumed.

- The weighting factor for the thyroid equivalent dose due to direct radiation is considered.

- Dose conversion factors are assumed according to ICRP119 [Ref. 14].

\subsubsection{Contribution accumulation in filters. Input data and assumptions}

All input data necessary for the definition of spectra by external cloud are necessary for determining activity and gamma energy spectra of radionuclides accumulated in the filters, noting that should generate new models to get the amount retained in filters.

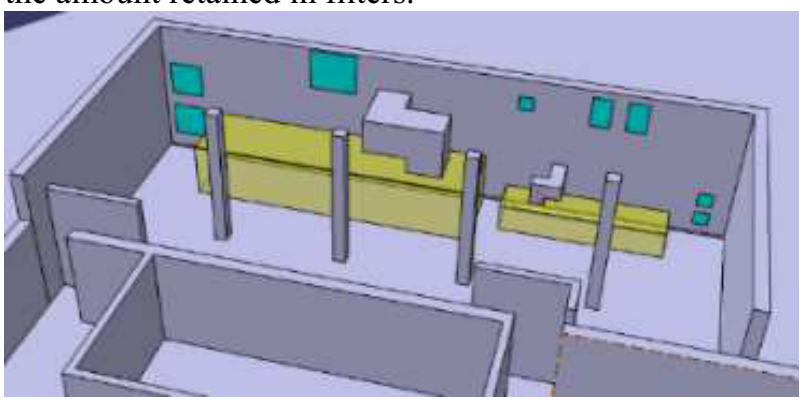

Fig. 4 Simplified Geometry Filtration Units

This activity retained in the filters thus becomes the source term for the Monte Carlo calculation, allowing 
the estimation of the thickness of shielding required or even the definition of the strategy for filter maintenance and management of the relevant waste.

The input data and particular hypothesis of this case would be:

- Volumetric flow HVAC system.

- Project drawings for the determination of the simplified geometry of the filters.

- Gamma energy spectra for characterization corresponding to the accumulation of radionuclides in the filtering units of CAGE volume source.

- All radionuclides will accumulate in the "virtual" filters, decaying in that filter.

- The activity generated by descendants is also considered in the accumulation of radionuclides in the filters.

- Inside the room HVAC, there are modeled only the two filter units, the walls corresponding to the labyrinths and walls defining this room.

- The outer dimensions of the filter units are modeled considering the geometric data of the drawings while the inner components are detailed by the typical constructive values.

- It has simplified model considering to assess the dose due to filters, are especially representative three zones: the interior of the room itself, protected areas through the mazes and inside the CAGE immediately behind the door in the adjoining area to HVAC room.

- Intensities resulting from the sum of the accumulation of isotopes in each time interval plus its corresponding decay daughters are considered. Thus, the fission products that are decaying in the filter at different time intervals are always considered.

\subsubsection{Contribution by direct radiation from containment. Input data and assumptions}

To carry out this simulation, it is proceeded in a similar way to that explained for the above cases.

Most of baseline data and hypotheses considered, coincide with those already set out throughout this document, so that only those that are particular to this model are mentioned:

- Drawings for determining the geometry of the simplified containment.

- It is considered conservatively that there is no leakage to the environment.

- Variance Reduction Techniques are used in the MCNP5 code because of the model dimensions and the shielding thicknesses that must be traversed.

\section{Simulations}

\subsection{Location}

Although the location of the buildings that house the CAGE obey multitude of conditions, among them is clearly identified, and so states the CSN in their design requirements, it should not be located in areas of predominant winds. If setting the building on one of these wind windows is mandatory, the design requirements in other areas will be influenced negatively, so each unit must locate the CAGE taking into account all factors, so that it can optimize the expenditures.

ARCON96 code utilization to determine the relative concentrations of radionuclides after a severe accident, allows to know the place where the concentration will be less. Especially sensitive to this situation would be the HVAC system, which may relax its demands in comparison to other place where concentrations were higher.

Once executed several cases, the $\mathrm{X} / \mathrm{Q}$ are determined at different time intervals, providing the necessary data in the next phase.

\subsubsection{Inner cloud}

Once introduced the input data and the assumptions, the implementation of the necessary simulations proceeds. The required results are TEDE and equivalent dose to the thyroid.

Throughout the project, there have been various adjustments that have enabled us to optimize the design of ventilation systems and sealing requirements of the building in general.

As an example may be mentioned that filtering recirculation is not required in the case of radiological accident, allowing cost optimization of the HVAC system.

\subsubsection{Outer cloud}

As previously stated, the radioactive cloud is a volumetric source term of gamma radiation, so to consider its contribution to the integral dose must be observed. The determination of this contribution can determine the thickness of the outer walls, in charge of providing the shielding necessary to maintain the habitability inside.

It is worth noting, that there may be cases where radiation limitation exceeds the limitation required from the seismic standpoint, prevailing over each other depending on the chosen location.

When it comes to characterize a source term, one must know its energy spectrum and the emission intensity $(\gamma / s)$ as to equal activity, the contribution to the dose will depend on the isotopes considered.

This characterization of the source term, along with the model geometry, the definition of materials and measuring points (tallies), define the "input" of MCNP5. This code allows the determination of the direct radiation dose at different points defined by the user. 


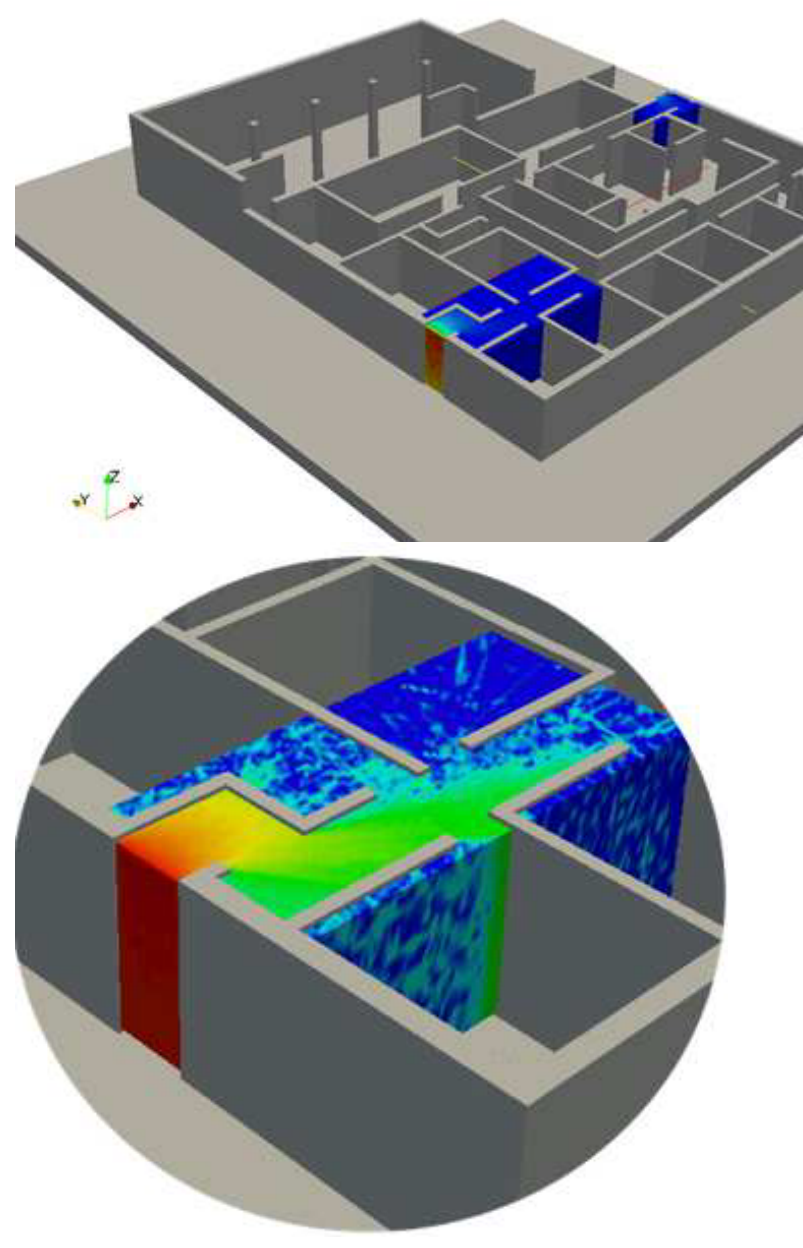

Fig. 5 Emergency exit detail

It should be noted that the outer cloud model has been paid to different hypotheses, finally opting for a semicylindrical representation of it that is considered conservative, as it homogenizes the limiting concentration at the selected location.

\subsubsection{Filtering units}

Similarly to the previous case, characterization of the source term is required, with the particularity that in this case, the concentration of radionuclides inside the filtration units increases over time, becoming a source term of great contribution to dose. The use of ORIGEN$\mathrm{S}$ code allows taking into account the daughters; isotopes that are added to the retained radionuclides themselves.

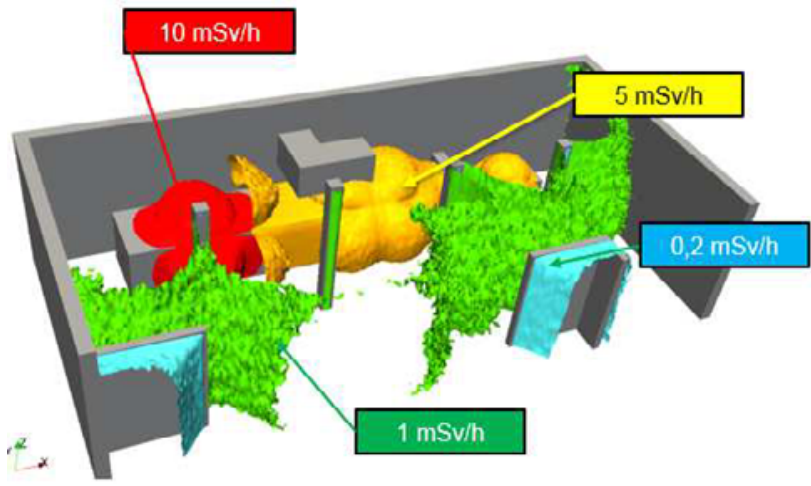

Fig. 6 Filtering Units iso-surface dose rates

Because of the magnitude of their contribution in this case the model is more detailed, simulating the materials of the filter housing and filter media. As shown in the previous figure, it has also been necessary to include labyrinthine accesses that reduce the dose.

Thanks to the results obtained by these simulations, the dimensions of the mazes and thickness of the separating wall of the room ventilation from rest of the building have been determined.

\subsubsection{Containment}

This simulation is similar to the outer cloud, with the proviso that the "cloud" is contained within the containment building.

Its contribution is found to be negligible after its evaluation.

\section{Conclusions}

Calculation codes selected for the development of this methodology are widely available, internationally used and validated in many studies. Therefore the robustness of the calculations depends primarily on the proper selection of input data and calculation assumptions.

The methodology disclosed herein allows for the modification of any of the parameters, so that it has developed a versatile method of radiological analysis.

Interaction with other areas' colleagues is key in the selection of (i.e. collaboration with HVAC specialists, civil design, etc.) the required valuable information.

On the other hand, knowledge of the design basis of the NPP where the CAGE is located it is essential to avoid incurring contradictions, being fundamental the existence of a fluid communication among all the interested parties.

It was required to characterize the HVAC room as an area of limited access to staff and managed directly by the Radiation Protection Department due to the dose rates obtained in the HVAC room.

After applying the methodology defined in this paper, the CAGE design is validated in compliance with the CSN requirements.

It is worth to point out that several iterations are required until the design of the building to imposed dose constraints has been tweaked. 


\section{References}

1. Consejo de Seguridad Nuclear. Instrucciones Técnicas Complementarias. ITC's a las Autorizaciones de Explotación. (2011).

2. Consejo de Seguridad Nuclear. Acta del Pleno 1.297, Anexo 4 Criterios de Evaluación del CAGE. (2013).

3. U.S. Nuclear Regulatory Commission. NUREG/CR6331 ARCON96 Atmospheric relative concentrations in building wakes. (1997).

4. U.S. Nuclear Regulatory Commission. NUREG/CR6604: RADTRAD A simplified model for RADionuclide Transport and Removal and Dose estimation. (1998).

5. OAK RIDGE NATIONAL LABORATORY. SCALE: A Comprehensive Modeling and Simulation Suite for Nuclear Safety Analysis and Design. (2011).

6. Los Alamos National Laboratory, MCNP - A General N-Particle Transport Code, Version 5 Volume I: Overview and Theory, X-5 Monte Carlo Team. April 2003. LA-UR-03-1987.

7. RG 1.23 Meteorological monitoring programs for nuclear power plants. Rev. 1, U.S. Nuclear Regulatory Commission, (2007).

8. RG 1.194 Atmospheric relative concentrations for control room radiological habitability assessments at Nuclear Power Plants. (2003).

9. U.S. Nuclear Regulatory Commission. NUREG1465: Accident Source Terms for Light-Water Nuclear Power Plants. (1995).

10. RG 1.195 Methods and assumptions for evaluating radiological consequences of Design Basis Accidents at Light-Water Nuclear Power Reactors. (2003).

11. EPA. FGR 11: Limit Values of Radionuclide Intake and Air concentration and Dose Conversion Factors for Inhalation, Submersion, and Ingestion. (1988).

12. EPA. FGR 12: External Exposure to Radionuclides in Air, Water and Soil.

13. RG 1.78: Evaluating the Habitability of a Nuclear Power Plant Control Room during a Postulated Hazardous Chemical Release. (2001).

14. ICRP. Compendium of Dose Coefficients based on ICRP Publication 60. ICRP Publication 119. Ann. ICRP 41(Suppl.), ICRP. (2012). 